\title{
Vía aérea "fisiológicamente" difícil: nuevo concepto a considerar - Caso clínico vía aérea difícil fisiológica
}

\author{
RICARDO URTUBIA V. 1,2, ALEJANDRO LILLO G. ${ }^{1}$, JOHNNY YÁÑEZ P. ${ }^{1}$, MANUEL ASTORGA A. ${ }^{\text {, }}$ \\ ORLANDO ESPARZA G. ${ }^{1}$, AXEL CAMOUSSEIGHT R. ${ }^{1}$, KARINNA QUILODRÁN V. ${ }^{1}$, JOSÉ PORCELL E. ${ }^{1}$
}

\begin{abstract}
Airway management in the ICU scenario is a real challenge due to instability imposed by illness severity. Some of these patients may also suffer from other diseases associated to difficult airway. Rheumatoid arthritis represents a special condition because not only can limit access to the hypopharynx, but also can hamper laryngoscopy. Among many options for difficult airway management, only a few can be safely applied to intubate the trachea while preserving physiologic stability in an emergency setting. We chose awake fiberoptic intubation as the first and safest approach for this combined difficult patient. This approach requires not only individual skills but also a plan in advance and a well coordinated team to be successful. A case is presented as an example of difficulties imposed by physiologic instability added to anatomic difficult.
\end{abstract}

\section{RESUMEN}

El manejo de la vía aérea en la UCI representa un desafío debido a la inestabilidad derivada de la severidad de la patología. Además, algunos de estos pacientes pueden presentar condiciones preexistentes asociadas a vía aérea difícil. La artritis reumatoide representa una condición especial debido a que no sólo limita el acceso a la hipofaringe, sino que también puede dificultar la laringoscopía. Entre las diferentes opciones para manejar la vía aérea, sólo algunas pueden ser aplicadas para intubar la tráquea de manera segura y al mismo tiempo preservar la estabilidad fisiológica en un contexto de urgencia. Este abordaje requiere no sólo habilidades individuales, sino que también trabajo en equipo coordinado y planificación. Se presenta un caso como ejemplo de dificultades impuestas por la inestabilidad fisiológica agregada a dificultad anatómica.
Key words: Intubation, laryngoscopy, intubation, intratracheal, airway management, rheumatoid, arthritis, respiratory insufficiency.

Palabras clave: Manejo de la vía aérea, intubación intratraqueal, artritis reumatoide, insuficiencia respiratoria, endoscopia.

Servicio de Anestesiología - Clínica Vespucio.

Facultad de Medicina, Escuela de Enfermería-Centro de Simulación, Universidad Finis Terrae. Correspondencia:

Dr. Ricardo Urtubia Valenzuela

E-mail: ricardo.urtubia@gmail.com

Los autores de este reporte de caso clínico declaran que ninguno tiene conflictos de intereses con los dispositivos mencionados. 


\section{Introducción}

$\mathrm{M}$ uchos de los pacientes que ingresan a unidades críticas requieren soporte ventilatorio invasivo, por lo que deben ser sometidos a intubación traqueal. Las alteraciones fisiológicas de estos pacientes representan un tipo de dificultad hasta el momento no considerada. Para el éxito de la intervención, estas requieren ser incluidas en el plan de manejo de la vía aérea. Se presenta un caso clínico para ilustrar las dificultades impuestas por la inestabilidad fisiológica agregada a dificultades anatómicas.

\section{Caso clínico}

Una paciente mujer de 55 años, obesa con un Índice de Masa Corporal (IMC) de 30,1, ingresó al Servicio de Urgencia con historia de tos, sensación febril, disnea y compromiso del estado general de 3 días de evolución. Tenía antecedente de hipotiroidismo y artritis reumatoide, diagnosticadas y en tratamiento hacía 5 meses. Al examen inicial destacaba una Presión Arterial (PA) de 106/77 mmHg, una Frecuencia Cardíaca (FC) de 125 l/min y una Frecuencia Respiratoria (FR) de 60/min, cianosis peribucal con Saturación parcial de Oxígeno (SpO2) de 60/min, cianosis peribucal con $\mathrm{SpO}_{2} 90 \%$, y temperatura de $38,4^{\circ} \mathrm{C}$. Se pesquisó neumopatía severa con insuficiencia respiratoria aguda, por lo que se decidió su ingreso en unidad de cuidados intensivos (UCI) para soporte ventilatorio, inicialmente no-invasivo (VNI).

Debido a que la paciente persistió inestable con signos de inminente agotamiento, se planteó intubarla y conectarla a ventilación mecánica invasiva. Para ello se solicitó evaluación anestesiológica destacando el antecedente de artritis reumatoide (AR). Comorbilidades tales como tabaquismo y patología cardiopulmonar eran negativos. En la evaluación dirigida de la vía aérea se pesquisaron los siguientes signos considerados predictores sugerentes de dificultad para la ventilación con máscara facial, laringoscopía-intubación y uso de dispositivos supraglóticos:

- Distancia interincisivos de $26 \mathrm{~mm}$.

- Micrognatia, con distancia arcada superior - inferior de $9 \mathrm{~mm}$.

- Signo de Mallampati grado 4.

- Flexo-extensión cervical de $30^{\circ}$, grado II.

- Test de mordida del labio superior grado 3.

Dado que la patología respiratoria aguda condicionaba nula tolerancia a un período de apnea y al decúbito dorsal, más la eventualidad de tener el estó- mago lleno, se decidió planificar una intubación con fibroscopio flexible (FBF) vigil bajo anestesia tópica (Plan A). Se explicó el procedimiento y las razones para efectuarlo a la paciente y se obtuvo consentimiento informado tanto para el procedimiento como también para la captura de imágenes del mismo.

Se disponía de un FBF Pentax ${ }^{\circledR}$ (Pentax UK Limited, Heron Drive, Langley, UK) de $31 \mathrm{~mm}$ de diámetro con canal de trabajo, una cánula VAMA ${ }^{\circledR}$ (AJL Ophtalmic SA, Miñano-Alava, España), un catéter epidural 16G para realizar técnica SAYGO ("spray-as-you-go"), lidocaína $2 \%$, Xylocaína spray $10 \%$, Lidocaína gel $4 \%$, y un tubo endotraqueal de $7,5 \mathrm{~mm}$ de Diámetro Interno (DI). Se instaló una cánula nasal a la paciente con flujo de $\mathrm{O}_{2}$ de $10 \mathrm{~L} / \mathrm{min}$ humidificado para oxigenación continua y se administró fentanilo $100 \mu \mathrm{g} \mathrm{IV}$ y atropina 0,4 mg IV. Se procedió a anestesiar el lumen traqueal mediante la instilación de lidocaína 1\% $40 \mathrm{mg}$ puncionando la membrana cricotiroidea con cánula venosa $22 \mathrm{G}$. Adicionalmente se bloqueó el glosofaríngeo con spray de Xylocaína y topicalización con lidocaína gel sobre el pilar palatogloso bilateral y la base de la lengua. Estos bloqueos fueron suficientes para que la paciente tolerara perfectamente la colocación de la cánula VAMA ${ }^{\circledR}$.

Con la paciente sentada y el operador frente a ella, se avanzó el FBF con un tubo traqueal enhebrado por el lumen de la cánula VAMA ${ }^{\circledR}$, hasta identificar la carina. Debido a que la diferencia de diámetro entre el FBF y el tubo traqueal era importante, se observó un atasco del tubo en el aritenoides derecho que, sin embargo, pudo resolverse con el giro del tubo en $90^{\circ}$ antihorario recomendado. No hubo evidencia de trauma en el procedimiento, el cual concluyó exitosamente.

\section{Discusión}

La intubación traqueal en el paciente crítico imprime diversos tipos de exigencias al equipo tratante: de tiempo por la urgencia, de competencias técnicas y no-técnicas, estrategias avanzadas, de trabajo en equipo y también derivado de la inestabilidad característica del paciente crítico ${ }^{1,2,3}$. Por esto es fácil comprender que la intubación de urgencia sea uno de los procedimientos con mayor riesgo en el manejo de estos pacientes y que se recomienden estrategias para mejorar su eficacia ${ }^{4,5}$.

Se ha descrito que las complicaciones asociadas a la intubación de urgencia en el paciente crítico pueden ser tanto de tipo mecánico como fisiológico $0^{6,7,8,9}$.

1. Las complicaciones mecánicas, descritas son: intubación esofágica (1,3 a 11\%), aspiración bron- 
quial (2 a 5,9\%), e intubación fallida (2,7\%). Adicionalmente, estos fenómenos mecánicos también afectan la incidencia de las complicaciones fisiológicas descritas a continuación, por lo que su ocurrencia tiene implicancias adicionales.

2. Las complicaciones fisiológicas que pueden observarse son: hipoxemia severa (4,7 a $26 \%)$ a pesar de preoxigenación, hipotensión severa (6 a $25 \%)$, bradicardia $(1,6 \%)$ y paro cardíaco $(1,8$ a $3 \%)$. Estas pueden ser producidas tanto por el efecto supresor del estímulo simpático de las drogas utilizadas para el manejo como por el efecto deletéreo que sobre la hemodinamia puede tener la ventilación a presión positiva ${ }^{10}$. Por esto es importante considerar también estos factores para definir el esquema que tendrá el menor impacto fisiológico posible ${ }^{11}$.

Mosier y cols ${ }^{12}$, han conceptualizado este fenómeno bajo el término "vía aérea fisiológicamente difícil". Esto se refiere a que la presencia de estas alteraciones fisiológicas representadas por: hipoxemia, hipotensión, acidosis metabólica severa y falla ventricular derecha, pueden constituir un tipo de dificultad que también puede conducir a complicaciones y que es necesario pesquisar y compensar antes de manejar la vía aérea ${ }^{13}$

En esta paciente se conjugaban la dificultad fisiológica dada por hipoxemia secundaria al fenómeno de shunt intrapulmonar, y la dificultad anatómica dada por la comorbilidad, constituyéndose el cuadro clínico en una dificultad mixta (anatómica y fisiológica).

Las recomendaciones sugeridas por Mosier y cols ${ }^{14}$ para manejar un caso como el presentado, son:

- Preoxigenación: Baillard y $\operatorname{cols}^{15}$, en un estudio con 53 pacientes con IRA hipoxémica, sugirieron que la VNI es más eficaz que los métodos tradicionales de preoxigenación con máscara facial. Recientemente, Jaber y $\operatorname{cols}^{16}$, describieron que la adición de oxigenación nasal de alto flujo (ONAF) a la VNI era mejor que la VNI sola.

- Oxigenación continua: El uso de cánulas nasales comunes con flujo de $\mathrm{O}_{2}$ de $15 \mathrm{~L} / \mathrm{min}$, han generado una interesante línea de investigación en favor de prolongar el tiempo seguro de apnea ${ }^{17,18}$. Sus resultados alentadores llevaron a la aplicación de la ONAF para este efecto y otros, incluyendo cirugía y procedimientos endoscópicos, también con experiencia favorable ${ }^{19,20}$.

Debido al riesgo elevado de regurgitación/aspiración, es necesario implementar una estrategia que lo reduzca. La clásica técnica de "intubación en secuencia rápida" se aplica en estos casos cuando no hay dificultad anticipada para el manejo de la vía aérea. Por el contrario, cuando se prevé dificultad, la recomendación universal es preservar la ventilación espontánea y los reflejos protectores de la vía aérea. Por tanto, en esta paciente crítica, el enfrentamiento escogido intentó respetar estos preceptos para ofrecer la mayor seguridad en el procedimiento.

La AR es causa de dificultad en el manejo de la vía aérea por varias razones:

- Limitación de la apertura oral por compromiso de la articulación temporomandibular (ATM). Formas más severas se asocian a micrognatia ${ }^{21}$.

- Laringoscopía difícil por compromiso artrítico aritenoideo, los cuales pueden estar edematosos y fijos en aducción $22,23,24$.

- Imposibilidad de lograr la posición de olfateo por reducción de la movilidad cervical ${ }^{25}$.

Por todo lo anteriormente expuesto, se consideró que el plan de manejo debía cumplir con los siguientes objetivos:

- Manejar la dificultad anatómica con fibroscopía flexible vigil bajo topicalización de la vía aérea conservando los reflejos protectores de la vía aérea ${ }^{26}$.

- Manejar la dificultad fisiológica preservando la ventilación espontánea (con técnica vigil, preoxigenación y oxigenación continua) y la hemodinamia manteniendo el tono simpático.

Adicionalmente, el plan debía incluir alternativas de manejo en caso de fracaso del plan inicial. Los planes alternativos a considerar en caso de falla fueron:

- Plan B: intubación traqueal bajo anestesia tópica con videolaringoscopio.

- Plan C: intubación fibroscópica a través de dispositivo supraglótico de $2^{\mathrm{a}}$ generación.

- Plan D: intubación retrógrada.

Descartamos la videolaringoscopía vigil debido a que la paciente no toleraba el decúbito dorsal y por una eventual laringoscopía difícil por la AR. También descartamos inicialmente la alternativa de utilizar un dispositivo supraglótico como conducto para una intubación fibroscópica, relegándola a un rol de rescate.

Una alternativa de sedoanalgesia en la UCI puede ser el uso de dexmedetomidina y/o ketamina, que brindan sedación adecuada y permiten mantener ventilación espontánea y la hemodinamia ${ }^{27,28}$.

Entre las limitaciones existentes en este caso, podemos citar disponer sólo de un fibroscopio muy fino 
para el diámetro del tubo que la paciente requería. Tampoco disponíamos de una mascarilla endoscópica, la cual hubiera sido una alternativa para mantener la VNI durante la fibroscopía/intubación ${ }^{29}$.

La vía aérea difícil, al estar influenciada por múltiples factores, tales como aquellos derivados del paciente (anatómicos y fisiológicos), aquellos que dependen del equipo humano involucrado (técnicos y no-técnicos) y también por factores ambientales (equipamiento, lugar), es un concepto difícil de precisar $^{30}$. Este caso, ocurrido fuera del Pabellón, con las limitaciones descritas y exigencia de eficacia y seguridad, demuestra que no sólo los elementos técnicos son esenciales sino que también los llamados "factores humanos" juegan un rol clave en la resolución de los problemas ${ }^{31}$.

\section{Referencias}

1. Urtubia R. Manejo de la vía aérea en el paciente crítico. En: Ugarte, Tomicic, Marks "Emergencias Médicas y Paciente Crítico", $2^{a}$ edición 2016, Editorial Mediterráneo. Capítulo 11, página 133.

2. Higgs A, Cook TM, McGrath BA. Airway management in the critically ill: the same but different Editorial. Br J Anaesth 2016;117 S1:i9-13.

3. Nolan JP, Kelly FE. Airway challenges in critical care. Anaesthesia 2011 Dec;66 Suppl 2:81-92.

4. Natt BS, Malo J, Hypes CD, Sakles JC, Mosier JM. Strategies to improve first attempt success at intubation in critically ill patients. Br J Anaesth 2016 Sep;117 Suppl 1:i60-8.

5. Mort TC. Emergency tracheal intubation: complications associated with repeated laryngoscopic attempts. Anesth Analg 2004 Aug;99(2):607-13.

6. Griesdale DE, Bosma TL, Kurth $T$, Isac G, Chittock DR. Complications of endotracheal intubation in the critically ill. Intensive Care Med 2008 Oct;34(10):1835-42.

7. Schwartz DE, Matthay MA, Cohen NH. Death and other complications of emergency airway management in critically ill adults. A prospective investigation of 297 tracheal intubations. Anesthesiology 1995 Feb;82(2):367-76.

8. Mort TC. The incidence and risk factors for cardiac arrest during emergency tracheal intubation: a justification for incorporating the ASA Guidelines in the remo- te location. J Clin Anesth 2004 Nov; 16(7):508-16.

9. Jaber $S$, Amraoui J, Lefrant JY, Arich C, Cohendy R, Landreau $L$ et al. Clinical practice and risk factors for immediate complications of endotracheal intubation in the intensive care unit: a prospective, multiple-center study. Crit Care Med 2006 Sep;34(9):2355-61.

10. Mort TC. Complications of emergency tracheal intubation: hemodynamic alterations-part I. J Intensive Care Med 2007 MayJun;22(3):157-65.

11. Mosier JM, Law JA. Airway management in the critically ill. Intensive Care Med 2014 May;40(5):727-9.

12. Mosier JM, Joshi R, Hypes $C$ Pacheco G, Valenzuela T, Sakles JC. The physiologically difficult airway. West J Emerg Med 2015 Dec;16(7):1109-17.

13. Myatra SN. Tracheal intubation in the ICU: life saving or life threatening? - Editorial. Bangladesh Crit Care J 2015;3(1):1.

14. Mosier JM, Hypes CD, Sakles JC. Understanding preoxygenation and apneic oxygenation during intubation in the critically ill.

15. Baillard C, Fosse JP, Sebbane $M$, Chanques $G$, Vincent $F$, Courouble $P$ et al. Noninvasive ventilation improves preoxygenation before intubation of hypoxic patients. Am J Respir Crit Care Med 2006 Jul;174(2):171-7.

16. Jaber S, Monnin M, Conseil M et al. Apnoeic oxygenation via high-flow nasal cannula combined with non invasive ventilation preoxygenation for intubation in hipoxaemic patients in the intensive care unit: single-centre, blinded, randomized, controlled OPTINIV trial. Intens Care Med; 2016.

17. Weingart SD, Levitan RM. Preoxygenation and prevention of desaturation during emergency airway management. Ann Emerg Med 2012 Mar;59(3):165-75.

18. Hayes-Bradley $C$, Lewis $A$, Burns $B$, Miller M. Efficacy of nasal cannula oxygen as a preoxygenation adjunct in energency airway management. Ann Emerg Med 2016 Aug;68(2):174-80.

19. Patel A, Nouraei SA. Transnasal Humidified Rapid-Insufflation Ventilatory Exchange (THRIVE): a physiological method of increasing apnoea time in patients with difficult airways. Anaesthesia 2015 Mar;70(3):323-9.

20. Ricard JD. Hazards of intubation in the ICU: role of nasal high flow oxygen therapy for preoxygenation and apneic oxygenation to prevent desaturation. Minerva Anestesiol 2016 Oct;82(10):1098-106.

21. Müller M, Pippi-Ludwig W. [Perioperative management of patients with rheumatoid arthritis]. Anaesthesist 2014 Nov;63(11):883-94.

22. Beirith SC, Ikino CM, Pereira IA. Laryngeal involvement in rheumatoid arthritis. Braz J Otorhinolaryngol 2013 MarApr;79(2):233-8.

23. Vetter TR. Acute airway obstruction due to arytenoiditis in a child with juvenile rheumatoid arthritis. Anesth Analg 1994 
Dec;79(6):1198-200.

24. Sato $S$, Asai T, Hashimoto $Y$, Arai T, Okuda Y. [Airway obstruction during attempts at fiberoptic intubation in an awake patient]. Masui 2014 May;63(5):548-51.

25. Reed AP. Evaluation and recognition of the difficult airway. In Benumof and Hagberg's Airway Management, 3rd Edition (2013), Chapter 9: 209-221.

26. Simmons ST, Schleich AR. Airway regional anesthesia for awake fiberoptic intubation. Reg Anesth Pain Med 2002 Mar-
Apr;27(2):180-92.

27. Scher CS, Gitlin MC. Dexmedetomidine and low-dose ketamine provide adequate sedation for awake fibreoptic intubation. Can J Anaesth 2003 JunJul;50(6):607-10.

28. Heidegger Th, Schnider ThW. "Awake" or "sedated": Safe bronchoscopic intubation of the difficult airway. Anesthesia Analgesia 2016.

29. Aoyama K, Yasunaga E, Takenaka I, Kadoya T, Sata T, Shigematsu A. Positive pressure ven- tilation during fibreoptic intubation: comparison of the laryngeal mask airway, intubating laryngeal mask and endoscopy mask techniques. Br J Anaesth 2002 Feb;88(2):246-54.

30. Huitink JM, Bouwman RA. The myth of the difficult airway: airway management revisited. Anaesthesia 2015 Mar;70(3):244-9.

31. Rutherford JS. Monitoring teamwork: a narrative review. Anaesthesia 2017 Jan;72 Suppl 1:84-94. 